\title{
MHD Simulation of Supernova Remnants
}

\author{
D. Wu ${ }^{1,2}$, M. F. Zhang ${ }^{1,3}$, S. S. Shan ${ }^{1,3}$ and W. W. Tian ${ }^{1,4}$ \\ ${ }^{1}$ Key Laboratory of Optical Astronomy, National Astronomical Observatories, \\ Chinese Academy of Sciences, Beijing 100012, China \\ email: wudan@bao.ac.cn \\ ${ }^{2}$ College of Information Science and Technology, Beijing Normal University, \\ Beijing 100875, China \\ ${ }^{3}$ School of Astronomy and Space Science, University of Chinese Academy of Sciences, \\ 19A Yuquan Road, Shijingshan District, Beijing 100049, China \\ ${ }^{4}$ Department of Physics \& Astronomy, University of Calgary, \\ Calgary, Alberta T2N 1N4, Canada
}

\begin{abstract}
We present some Magnetohydrodynamic (MHD) simulations for supernova remnants (SNRs), which hints the thicknesses of SNRs' shells are likely related to the density and density distribution of surrounding interstellar medium (ISM). The simulations show clear formation and evolution of reverse shocks. In addition, we find that stellar winds can blow a bubble around the progenitor stars then lead to weaker radio emission at the center of SNRs.
\end{abstract}

Keywords. Methods: numerical, ISM: supernova remnants, MHD

\section{Introduction}

Supernova shock interacts with the ISM then generate SNRs. There are many unsolved puzzles about SNRs, and the key to unlock these is to figure out the evolution of SNRs. Full of randomness, the evolution of SNRs is not only dependent on the progenitor, but also influenced by the surrounding ISM. But, MHD simulations can help us understand such a complicated process.

The shells of different SNRs usually have different thicknesses, which are determined by many conditions, such as the ejecta mass, explosion kinetic energy and the density of ISM. And they can be also influenced by some random parameters, such as surrounding molecular clouds (MCs) and even the shapes of MCs (Koo \& Moon 1997). In the simulations, we adjust these initial conditions to study ISM' contributions to the thickness. To get more reasonable initial conditions, we take the stellar wind of progenitor star, magnetic field and the nearby MCs into consideration. Assuming the radio flux density of SNRs totally originates from synchrotron emission, we convert the simulation results to radio flux density maps and smooth them in terms of the resolution of real observation, so that we can compare the simulations with observations easily.

\section{Simulation model and results}

Ignoring gravitation and dissipative effects, we can get an ideal conservation equation set, including mass, energy, momentum and magnetic conservations. On the basis of the set, we perform the simulation on PLUTO Mignone et al. (2007), a code used to study MHD. We assume a supernova with an explosion kinetic energy of $2 \times 10^{51}$ erg and an ejecta mass of $15 \mathrm{M}_{\odot}$. The hydrogen atom number densities of ISM are set to $2 \mathrm{~cm}^{-3}$ and $20 \mathrm{~cm}^{-3}$. The initial radius is set to $1 \mathrm{pc}$, based on which the initial velocity and 

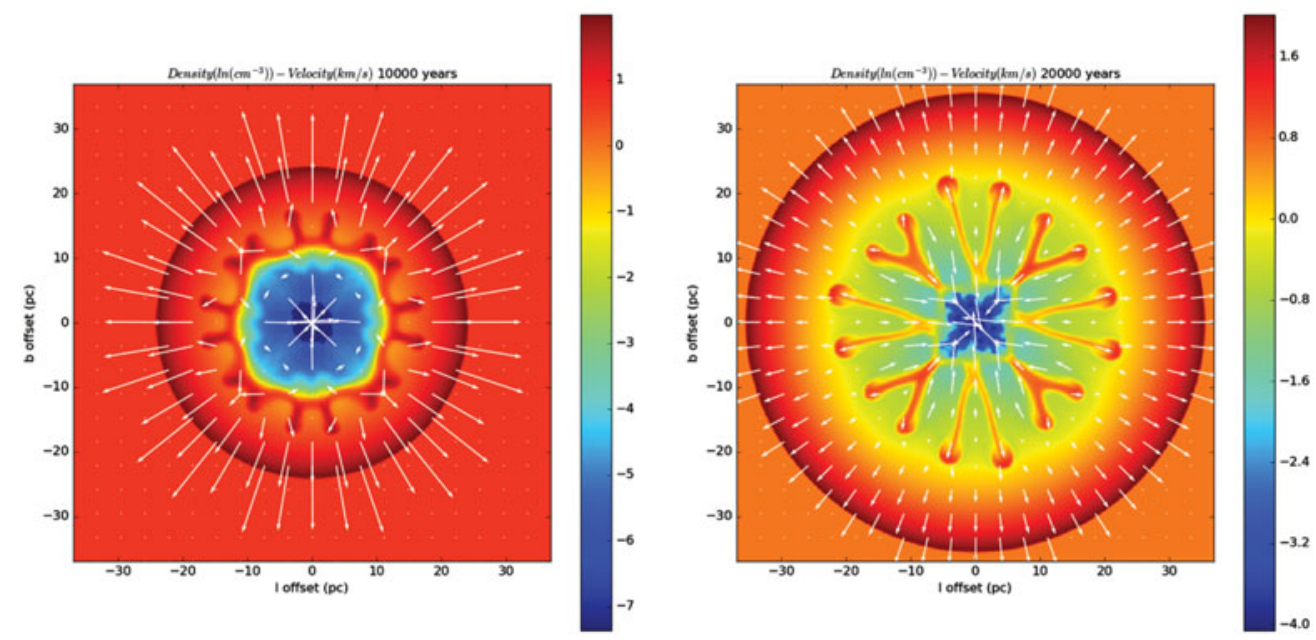

Figure 1. The density-velocity maps. The top tow images show the simulated results after 10000, 20000 years with a initial ISM density of $2 \mathrm{~cm}^{-3}$. White arrows stand for velocities.
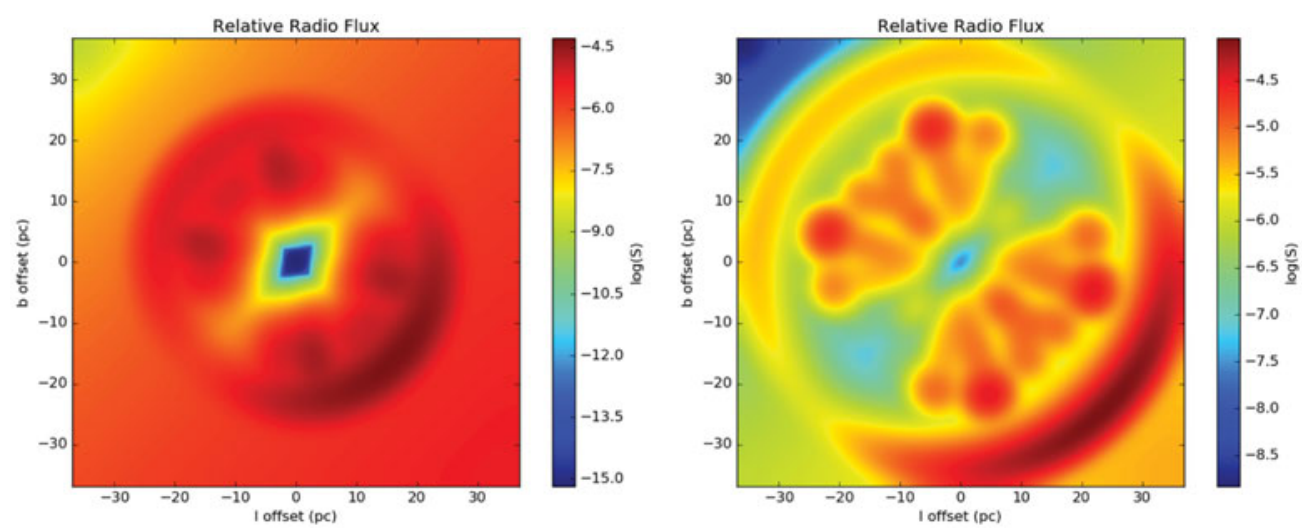

Figure 2. Relative radio flux maps. The images shows the corresponding relative radio flux in Fig. 1.

density can be estimated by the work of Leahy \& Williams (2017). To simulate a one-side SNR, we add a magnetic gradient increasing from upper left to lower right. The magnetic field in the explosion center is set to $9 \mu \mathrm{G}$ and the magnetic field gradient is $0.1 \mu \mathrm{G} / \mathrm{pc}$. The relative radio flux is calculated by $i(\nu)=C \rho B_{\perp}^{\beta+1} \nu^{-\beta}$ (Orlando et al. 2007). In the simulation, $\beta$ is set to 0.25 . If we use a telescope with a resolution of $4^{\prime}$ observe our simulated SNRs at about $4 \mathrm{kpc}$, the observed images should be the simulation results smoothed by a gaussian function with a standard deviation of 14 referring to the number of pixels.

Fig. 1 shows the simulated density-velocity maps at different ages. Fig. 2 shows the corresponding relative radio flux maps. In Fig. 3 and Fig. 4 , we adopt a power law density distribution $N(\rho)=N_{0} \rho^{-\alpha}$, in which $\mathrm{N}$ is the pixel numbers at different densities $\alpha$ is set to 2.4 , i.e., low-density regions are more than high-density regions. Fig. 3 shows simulated density-velocity maps at $20 \mathrm{~cm}^{-3}$, while Fig. 4 shows the relative radio flux maps at $2 \mathrm{~cm}^{-3}$.

To understand the influence of stellar winds, we use another parameter set, in which the explosion kinetic energy is $0.8 \times 10^{51} \mathrm{erg}$, the density of ISM is about $20 \mathrm{~cm}^{-3}$. The 

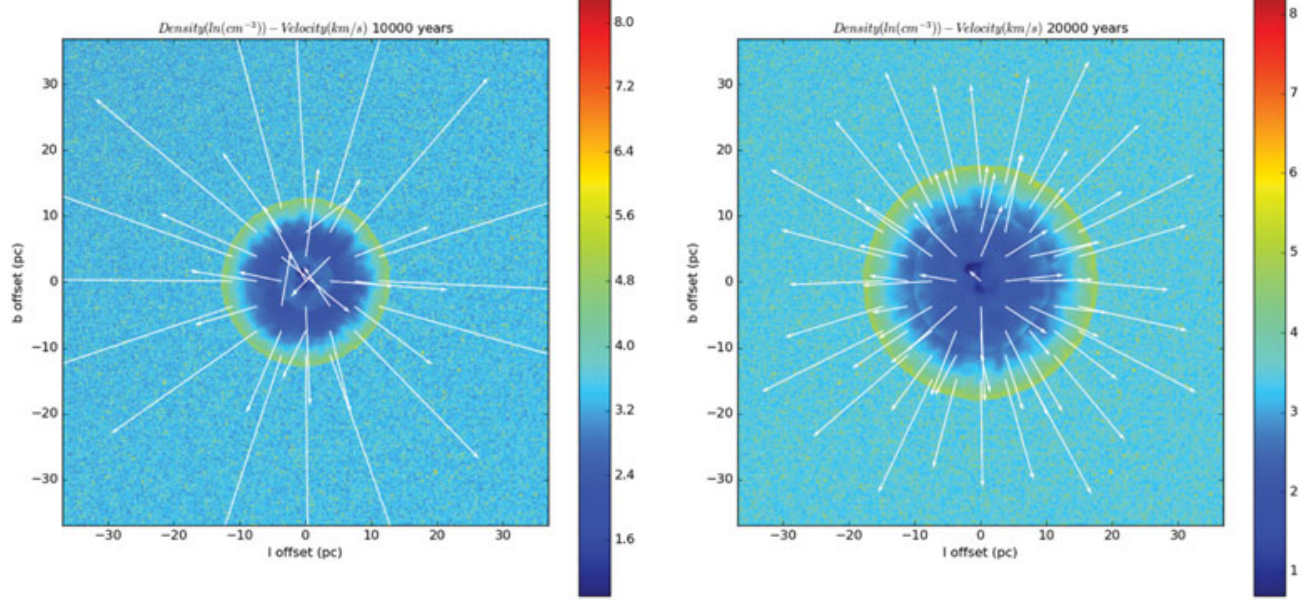

Figure 3. The density-velocity maps. The two images show the simulated results after 10000, 20000 years with a initial ISM density about $20 \mathrm{~cm}^{-3}$. White arrows stand for velocities.
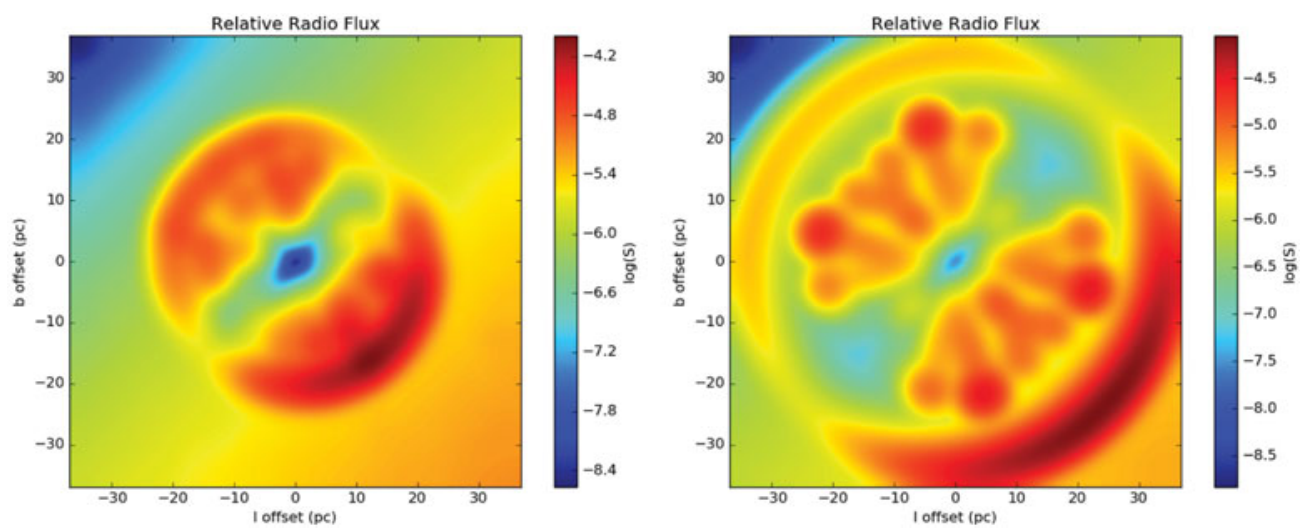

Figure 4. Relative radio flux maps. The two images show the radio flux map after 10000, 20000 years with a initial ISM density about $2 \mathrm{~cm}^{-3}$.

density distribution is set based on the background CO data from GRS (Jackson et al. 2006). The stellar winds model is the standard model in Lamers \& Cassinelli (1999). We simulate a progenitor star blowing stellar winds in one million years and use the final density distribution as the initial density. To study more details, we get simulation results after 30000 years and do not smooth them. In Fig. 5, we show the results with and without stellar winds after 30000 years.

\section{Discussion}

In Fig. 1, we can see the velocity changes direction towards the explosion center at 10000 years, which indicates the formation of reverse shocks. In Fig. 2, there are two shells and obvious inside structures at $2 \mathrm{~cm}^{-3}$ maps. If the flux density of darker shell is lower than the sensitivity of a telescope, it is not visible, then we can only see one shell. In addition, these inside structures will make the shells thicker. Comparing it with Fig. 1, it is reasonable to guess reverse shocks and instabilities lead to these inside structures. 

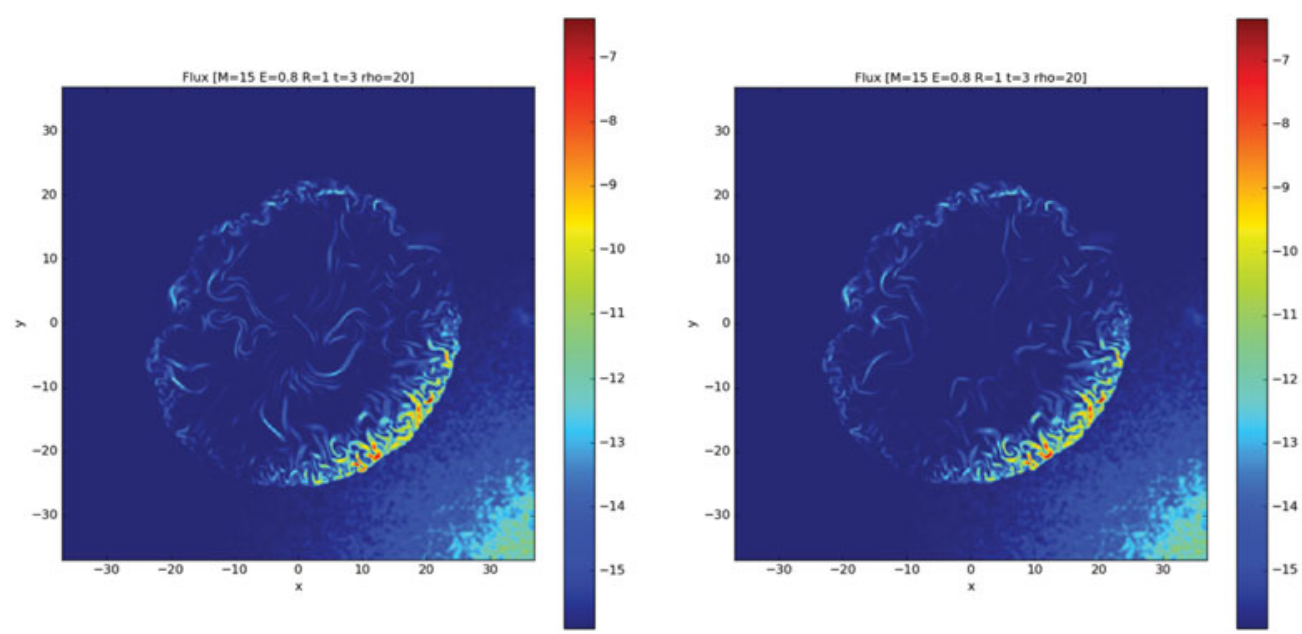

Figure 5. Relative radio flux maps without smoothing. The two pictures are both the flux at same ISM background. The right one takes the stellar winds into consideration.

In Fig. 3, reverse shocks become secondary forward shocks after they converge to the explosion center and form two layers with the main forward shocks. However, we ignore the dissipative effects in the simulation, if not, the secondary forward shocks will be much weaker. The inner layer, generated by reverse shocks, is low-density and hightemperature, so it is possible to radiate at X-ray. If it is real, then the phenomenon can be used to explain that the X-ray sizes of SNRs are usually less than radio sizes. The inner structures are easier to be distinguished from outer layers in Fig. 4. Comparing Fig. 4 with Fig. 2, we think the thickness of shells is also related to the density distribution.

Fig. 5 indicates the stellar winds of progenitors can only make the central small regions weaker and more uniform at radio wavelength during the evolution of SNRs. So the radio images of SNRs usually have a weak center and thick shells. In Fig. 5, we can see the diagonally asymmetric structure like Fig. 2 and Fig. 4 . This is entirely dependent on the magnetic field gradient. Although strong magnetic field can obstruct the shocks and make the ISM denser, the magnetic field here is so weak that such an effect can be ignored.

We will apply the simulations to some observed SNRs, and further test our conclusion.

\section{References}

Denis, A. Leahy \& Jacqueline, Williams 2017, AJ, 153, 239

Jackson, J. M., Rathborne, J. M., Shah, R. Y., Simon, R., Bania, T. M., Clemens, D. P., Chambers, E. T., Johnson, A. M., Dormody, M., Lavoie, R., \& Heyer, M. H. 2006 ApJS, 163,145

Jun, B.-I. \& Norman, M. L. 1996, ApJ, 465, 800

Koo, B.-C. \& Moon, D.-S. 1997, ApJ, 475, 194

Lamers, H. J. G. L. M. \& Cassinelli, J. P. 1999, Introduction to Stellar Winds

Mignone, A., Bodo, G., Massaglia, S., Matsakos, T., Tesileanu, O., Zanni, C., \& Ferrari, A. 2007, ApJS, 170, 228

Orlando, S., Bocchino, F., Reale, F., Peres, G., \& Petruk, O. 2007, A\& A, 470, 927 